\title{
Malnutrition among elderly a multifactorial condition to flourish: Evidence from a cross-sectional study
}

\author{
Ishfaq Rashid ${ }^{\mathrm{a}}$, Pramil Tiwari ${ }^{\mathrm{a}, *}$, Sarabmeet Singh Lehl ${ }^{\mathrm{b}}$ \\ ${ }^{a}$ Department of Pharmacy Practice, National Institute of Pharmaceutical Education and Research (NIPER), S.A.S. Nagar, Punjab, 160062, India \\ ${ }^{\mathrm{b}}$ Department of General Medicine, Government Medical College, and Hospital (GMCH), Chandigarh, India
}

\section{A R T I C L E I N F O}

\section{Keywords:}

Elderly

Malnutrition

Mini nutritional assessment tool

Risk factors

\begin{abstract}
A B S T R A C T
Aim: The negative outcomes like an increased risk of falling, anemia, immune dysregulation, impaired cognitive status, reduced muscle function, increased morbidity and mortality associated with malnutrition are believed to affect the economy of the country in the future years. In this backdrop, this study aims to evaluate the nutritional status and different risk factors associated with malnutrition among elderly patients at a tertiary care public teaching hospital.

Methods: This questionnaire-based study utilized the "Mini Nutritional Assessment-Short Form" tool to evaluate the nutritional status of elderly inpatients (aged 60 years or more). The data obtained from 235 participants reporting in the medicine department is presented. Based upon MNA-SF tool parameters, a well-determined score was obtained and on the basis of scores, the patients were categorized into "normal" (12-14), "at risk of malnutrition" (8-11 points) and "malnourished" (0-7 points). Multinomial logistic regression was performed to study the influence of different determinants on the nutritional status among elderly inpatients.

Results: The studied sample had a high frequency of $50.2 \%$ for the patients who are "at risk of malnutrition" and $46.4 \%$ of patients were "malnourished". Based on the evaluated scores of MNA-SF tool, this study also confirms that the mobility functions, the decline in food intake and weight loss are major risk factors for malnutrition. Conclusions: A very high prevalence of malnutrition was observed, in consonance with the previous reports. The findings also indicate that malnutrition is a multifactorial condition mostly associated with mobility dysfunction followed by somatic factors and socio-demographic factors. Further studies are required to map the risk factors in order to avoid the associated negative outcomes.
\end{abstract}

\section{Introduction}

Nutritional status assessment is a comprehensive evaluation to understand changes in human physiology. Poor nutritional status and malnutrition in the elderly population are important areas of concern. Malnutrition contributes to a progressive decline in health, an increased risk of falling, anemia, immune dysregulation, impaired cognitive status, reduced muscle function, increased utilization of health care services, premature institutionalization, and increased morbidity and mortality. ${ }^{1}$ Malnutrition is also associated with poor rehabilitative outcomes as well as worse functional performance. ${ }^{2}$ In India, the problem of the health of the elderly is compounded by poor nutrition together with medical issues, including both communicable and noncommunicable diseases.

Malnutrition has been shown to increase during hospitalization among elderly patients. ${ }^{3}$ The risk factors of malnutrition are extremely varied and they can be divided into three main types: medical factors like poor appetite, weight loss, mobility dysfunction, diseases, drug interactions, lifestyle \& social factors like lack of knowledge about food, cooking or nutrition, isolation, poverty and inability to shop or prepare food and psychological factors like confusion, dementia, depression, bereavement or anxiety. ${ }^{4}$ The most common risk factors associated with malnutrition are poor appetite, weight loss, mobility, abnormal body mass index, psychological stress or acute diseases and neuropsychological problems. Poor appetite is probably the major cause of malnutrition and is mediated by a variety of factors like loss of taste and smell, dysphagia, and poor dentition. Stroke patients with swallowing problems had a worse nutritional status compared with those with no swallowing problem. ${ }^{5}$ Many researchers have investigated the relationship between cognition and nutrition. They have reported that the feeding and alimentation skills regress gradually because of cognitive and physical deterioration; uncontrolled weight loss is almost

\footnotetext{
* Corresponding author. Department of Pharmacy Practice, NIPER SAS Nagar, Mohali, Punjab, 160062, India.

E-mail address: ptiwari@niper.ac.in (P. Tiwari).
} 
inevitable in later stages, despite quality of care. ${ }^{6} 50 \%$ of patients with Alzheimer's disease lose the ability to feed themselves eight years after diagnosis. ${ }^{7}$ Altered food intake is a symptom of depression and several studies have provided evidence to suggest that this is a comparatively common cause of weight loss and malnutrition in the elderly. ${ }^{8-10}$

Nutritional assessment is an in-depth evaluation of both objective and subjective data related to an individual's food and nutrient intake, lifestyle and medical history. There are several tests used to assess malnutrition in the elderly like MNA-SF tool, elderly nutritional risk index (GNRI) and Barthel Index assessment to evaluate functional status. The Mini Nutritional Assessment-Short Form (MNA-SF) tool is a validated and commonly used tool. It has high sensitivity and specificity and is effective for evaluating the nutritional status of elderly persons living in different settings. ${ }^{11,12}$ The MNA-SF tool, a single and rapid nutrition assessment tool, was developed to evaluate nutrition status as part of the standard evaluation of elderly patients in clinics, nursing homes, hospitals, or among those who are otherwise frail. It was designed to meet the specifications such as reliability, clearly defined thresholds, compatibility with the skills of a generalist assessor, the minimal opportunity for bias introduced by the data collector, acceptability by patients, and low cost. ${ }^{13}$

The aim of the nutritional assessment is to identify individuals or population groups at risk of becoming malnourished and to identify individuals or population groups who are malnourished. This study has evaluated the nutritional status, different risk factors associated with malnutrition and the influence of different determinants on the nutritional status among elderly inpatients at a tertiary care public teaching hospital.

\section{Materials and methods}

\subsection{Study design, sample, and setting}

This prospective cross-sectional study was conducted to evaluate the nutritional status and to study the influence of different determinants on nutritional status among elderly inpatients hospitalized with different illnesses. The patients, either sex and age 60 years or more, were enrolled from the selected inpatient departments (IPDs) of the General Medicine at a tertiary care public teaching hospital. The patients were then rated using the MNA-SF tool. Prior to data collection, a pilot study on the mini nutritional assessment scale was performed in order to minimize the data collection bias.

\subsection{Data collection procedure}

This study was carried out in full compliance with the ethical standards provided by Indian Council of Medical Research to carry out such study. The experimental protocol had the approval from the respective "research committee" and "ethics committee" of the concerned hospital and mentor institute. The verbal and written explanation of the study was performed; and, the written informed document from patients or patient's legal guardians or caretakers prior to enrolling subjects in the study was obtained.

The patients with age 60 year or above, either sex, with one or more concurrent diseases, receiving one or multiple drugs and willing to participate were included. The patients with incomplete documentation and completely immobilized patients and/or unable to answer the questions were excluded from the study. All the relevant information was collected from the patient record file and a patient interview was conducted for each patient in the study. Patient record file information included demographic characteristics (age, gender, body weight, and height), co-morbidities, diagnosis, and drugs prescribed. All this information was collected in a standard case record form. The patients were not subjected to any harm during this study and there was no direct benefit to them. Patient's rights were respected and the personal health information provided by the patient was confidential and in no circumstances, it was disclosed to anyone.

\subsection{Mini nutritional assessment - sort form (MNA-SF) tool}

The MNA-SF tool consists of six parameters i.e. food intake $(0,1$, or 2 points), weight loss $(0,1,2$, or 3 points), mobility ( 0,1 , or 2 points), psychological stress ( 0 or 2 points), neuropsychological problems $(0,1$, or 2 points), and body mass index ( $0,1,2$, or 3 points). Based upon the inputs on these parameters, a well-determined score was obtained for all patients. On the basis of these scores, the patients were categorized "malnourished" (0-7points), "at a risk of malnutrition" (8-11 points) and "normal nutritional status" (12-14 points). The frequencies of malnutrition risk factors were calculated based on their individual scores for each parameter using the MNA-SF tool. The individual items/ parameters i.e. mobility, and neuropsychological status were evaluated by the help of predetermined scores of their sub-parameters.

The mobility parameter was assessed by using three sub-parameters like the patients being "bed or chair bound", patients "able to go out of bed/chair but doesn't go out" and patients "goes out".

The neuropsychological parameter was assessed by using three subparameters such as the patients having "severe dementia and depression", patients with "mild dementia" and the patients having "no psychological problem".

Permission to use the Mini Nutritional Assessment tool for evaluating nutritional status among elderly patients was obtained from the developer. This is a prevalidated tool. ${ }^{13}$

\subsection{Data analysis}

All the data obtained during the study was organized into a spreadsheet. The descriptors of central tendency and variations were computed using Microsoft Excel ${ }^{\circledR}$ and Free version of SPSS. The results were presented in percentages and averages were supported with the Standard Error of Mean (SEM).

Multinomial logistic regression was performed in order to study the influence of different determinants like food habits, residence, socioeconomic status, decline in food intake, patients using walking aids, high fall risk drugs (HFRDs), urinary incontinence or urgency, and patients with more than three co-morbidities on nutritional status among elderly patients. Strength of multinomial logistic regression relationship was evaluated by using pseudo R square. In this case, the Cox and Snell R Square and the Nagelkerke R square value was used, which provides an indication of the amount of variation in the dependent variable.

The overall classification accuracy in the predictive table was used to evaluate the utility of the logistic model or to characterize the model as useful. The likelihood ratio test was used to evaluate the overall relationship between an independent variable and a dependent variable. $\mathrm{P}<0.05$ was taken to indicate statistical significance.

\section{Results}

In this cross-sectional study, 246 elderly patients were screened. Based on exclusion criteria, 11 patients were excluded from this study (4 with incomplete information and 7 patients not willing to participate in the study). Therefore, the results are based on data obtained from 235 patients. The average age of the patients was found to be $68.2 \pm 0.4$ years (range of 60-92 years).

Sample characteristics: A Shapiro-Wilk's test and a visual inspection of histograms, normal Q-Q plots and box plots showed that the mini nutritional assessment scores were approximately normally distributed for both males and females, with a skewness of $-0.207(\mathrm{SE}=0.220)$ and kurtosis of -0.065 (SE $=0.437$ ) for the females and a skewness of $0.139(\mathrm{SE}=0.226)$ and kurtosis of $-0.479(\mathrm{SE}=0.449)$ for the males. Thus the results are presented with mean.

Of 235,114 patients were male and 121 were female $(48.5 \%$ and 
$51.5 \%$ ). The average age of male patients was $68.8 \pm 0.6$ years and $67.7 \pm 0.5$ years for female patients; and, the difference was statistically insignificant.

The patients were classified into three categories "young-old" age group (60-69 yrs), "middle-old" age group (70-79 yrs) and "very-old" age group ( $80 \mathrm{yrs}$ or more). ${ }^{14} 130$ patients were "young-old", 85 patients were "middle-old" and 20 patients were "very-old".

The most common diagnoses of the patients enrolled were diabetes mellitus, hypertension, CKD, chronic liver disease, acute pancreatitis, coronary artery disease, COPD, Parkinson's disease and cholelithiasis. The number of diagnoses varied from 1 to 5 in these patients. 166 patients were found to have two or more diagnosis.

\subsection{Nutritional status evaluation}

A total of 235 patients were evaluated for nutritional status by using MNA-SF tool which is as under,

a Malnutrition: 109 patients were found to fall under the "malnutrition" category. 58 were females and 51 males. Of this 58 were "young-old", 43 were "middle-old" and 8 were "very-old". The average age of these patients was $68.5 \pm 0.6$ years.

b At Risk of Malnutrition: 118 patients were found to be "at risk of malnutrition". 61 females and 57 males. Out of 118, 65 were "young-old", 41 were "middle-old" and 12 were "very-old". The average age of these patients was $68.2 \pm 0.6$ years.

c Normal Nutrition: 8 patients were found to lie under "normal nutrition" category. 2 were females and 6 males. 7 patients belonged to the "young-old" group and 1 belonged to "middle-old" group. The average age of these patients was $63.0 \pm 1.0$ years.

The evaluation of nutritional status showed that 176 patients had vegetarian food habits and 81 reported consumption of alcohol (Table 1).

Various malnutrition risk factors have been identified like mobility functions, decline in food intake, weight loss, psychological stress, neuropsychological problems and body mass index. The frequency of malnutrition risk factors was calculated on the basis of their individual scores using MNA-SF tool (Fig. 1). The parameter that scored least was the largest contributor of malnutrition on the basis MNA-SF tool, as the score of "malnourished" category is (0-7 points).

Various somatic risk factors associated with malnutrition such as patients experienced fall within 6 months before admission, patients

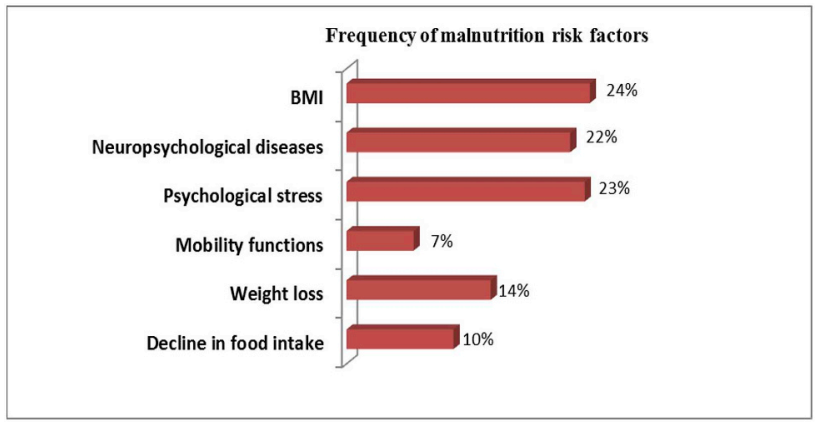

Fig. 1. Frequency of malnutrition risk factors.

receiving high fall risk drugs (HFRDs), patients receiving more than 6 medications, patients with urinary incontinence or urgency, patients required walking aids and patients having 3 or more comorbidities were also evaluated. $40 \%$ of patients using walking aids were found to be malnourished (Fig. 2).

The overall relationship between the different determinants and nutritional status was evaluated by performing multinomial logistic regression analysis (Table 2). The amount of variation in the dependent variable is described as pseudo $\mathrm{R}$ square. Cox and Snell $\mathrm{R}$ and Nagelkerke $\mathrm{R}$ square values were found to be 0.541 and 0.672 respectively. This indicates that $54 \%-67 \%$ of the variability is explained by these variables used in the model. The overall predictive accuracy for the present model is $79.1 \%$, suggesting that the model was useful.

\section{Discussion}

The results of this study are based upon data obtained from a set of 235 patients admitted to the inpatient departments of the General Medicine at a tertiary care public teaching hospital. The sample had a fair uniformity with respect to gender.

130 patients were "young-old", 85 patients were "middle-old" and 20 patients were "very-old". The reason for the larger number of patients in "young-old" and "middle-old" age group could be that the patients in this group suffer from a variety of acute and chronic illnesses that demand hospitalization.

In this study, the prevalence of malnutrition was found to be $46.4 \%$ which is higher as compared to the report from rural Tamil $\mathrm{Nadu}^{15}$ wherein $14 \%$ of the elderly patients were found to be malnourished. Agarwalla et al., ${ }^{16}$ Ferdous et al., ${ }^{17}$ and Saka et al. ${ }^{18}$ also had reported

Table 1

Nutritional status assessment based on Socio-demographic details.

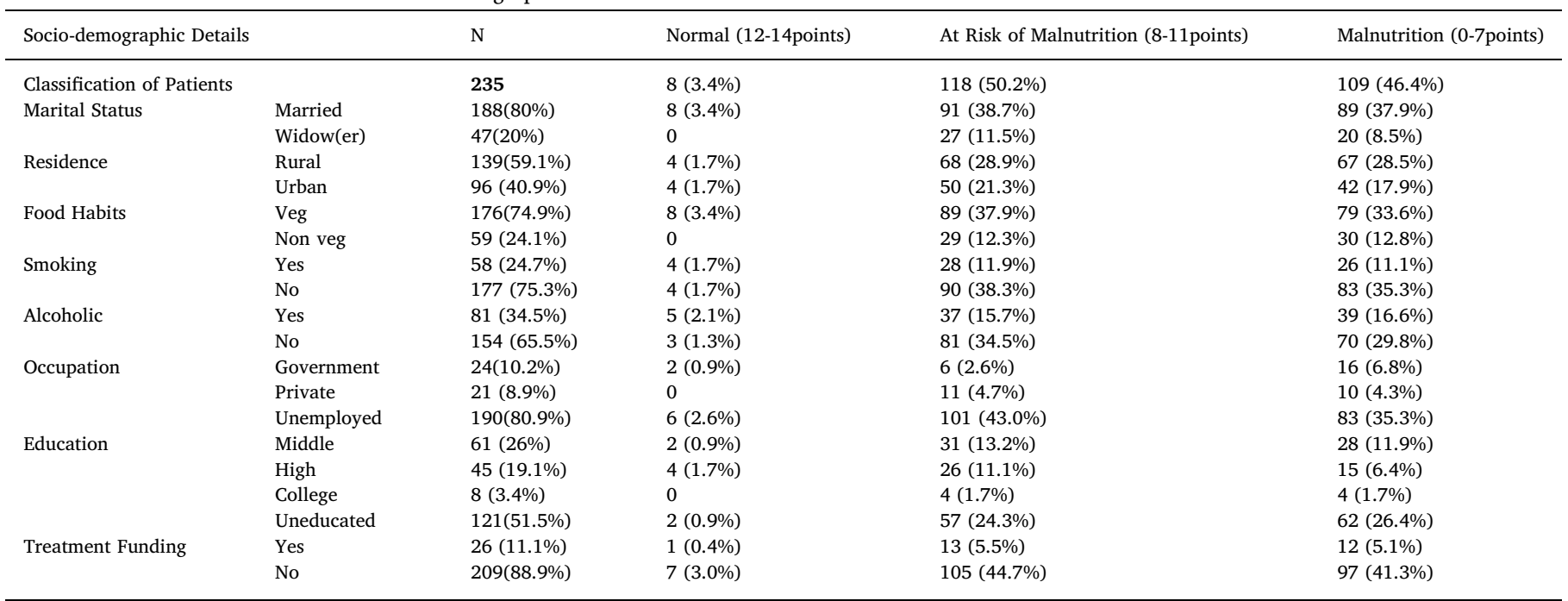




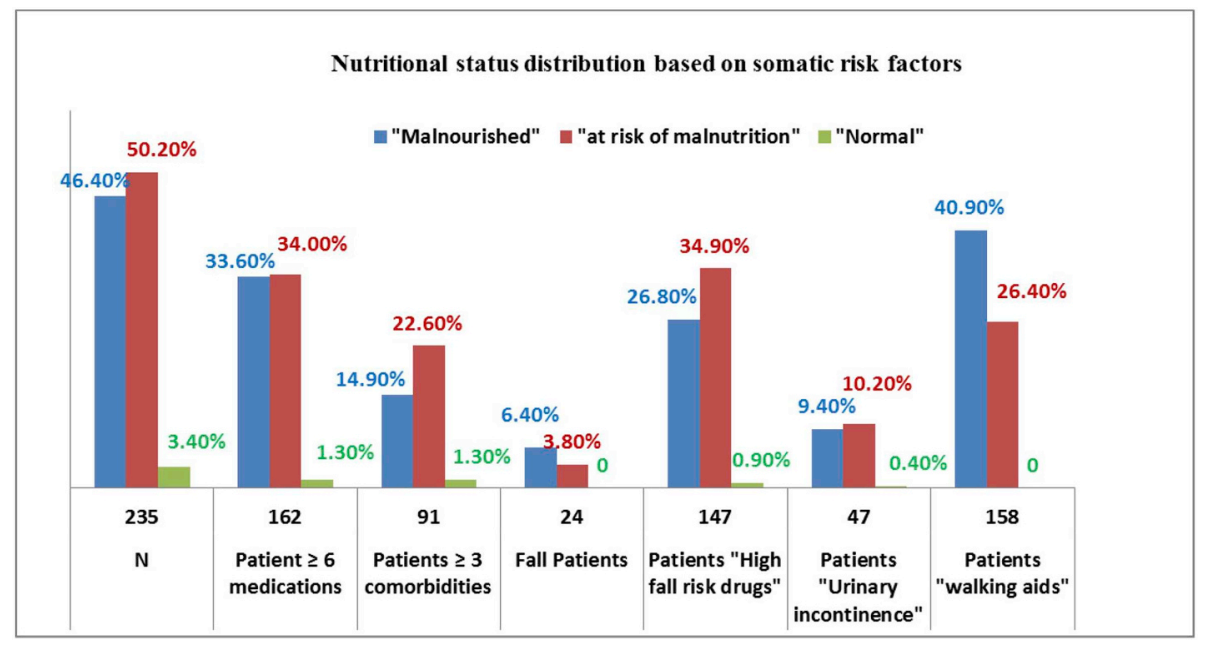

Fig. 2. Nutritional status distribution based on somatic risk factors.

Table 2

Overall relationship between different variables and nutritional status.

\begin{tabular}{llll}
\hline \multirow{2}{*}{ Effect } & \multicolumn{2}{l}{ Likelihood Ratio Tests } \\
\cline { 2 - 4 } & Chi-Square & df & P value \\
\hline Intercept & 0.000 & 0 & \\
age & 6.313 & 2 & $\mathbf{0 . 0 4 3}$ \\
Marital status & 3.727 & 2 & 0.155 \\
Residence & 7.352 & 2 & $\mathbf{0 . 0 2 5}$ \\
Food Habits & 11.413 & 2 & $\mathbf{0 . 0 0 3}$ \\
Smoking & 1.414 & 2 & 0.493 \\
Alcohol use & 11.502 & 2 & $\mathbf{0 . 0 0 3}$ \\
Occupation & 16.808 & 4 & $\mathbf{0 . 0 0 2}$ \\
Educational status & 19.168 & 6 & $\mathbf{0 . 0 0 4}$ \\
Treatment funding & 1.717 & 2 & 0.424 \\
Decline in food intake & 45.637 & 4 & $\mathbf{0 . 0 0 0}$ \\
Patients using walking aids & 67.160 & 4 & $\mathbf{0 . 0 0 0}$ \\
Urinary incontinence and urgency & 9.612 & 2 & $\mathbf{0 . 0 0 8}$ \\
High Fall Risk Drugs & 11.294 & 2 & $\mathbf{0 . 0 0 4}$ \\
Pseudo R-Square & & & \\
Cox and Snell & & 0.541 & \\
Nagelkerke & & 0.672 & \\
\hline
\end{tabular}

similar results. However, Anuradha et al. ${ }^{19}$ have reported that $51.67 \%$ of patients were malnourished and Saeidlou et al. in a nursing home in Iran $^{20}$ observed that a considerably higher percentage of $49.6 \%$ of the elderly were malnourished. This could be due to either the difference in profiling or characteristics of the study population.

In this study, $50.2 \%$ of the participants were found to be "at a risk of malnutrition" which is higher as compared to the study reported in Rajasthan ${ }^{21}$ who have reported $46 \%$ of prevalence, while comparing to the study reported in Assam ${ }^{16}$ the rate of prevalence was found to be $55 \%$ which is quite higher than this study, it may be either due to regional differences, or lifestyle. The prevalence of another study reported in Pakistan was found to be (42.10\%) smaller than this study, it may be due to higher sample size (380). ${ }^{22}$ This study also revealed that the prevalence of "malnutrition" and "risk of malnutrition" was almost the same in either gender.

To study the relationship of different determinants with nutritional status by performing multinomial logistic regression analysis, a significant relationship was observed between age, Food habits, alcoholic status, residence, Occupation, Education and nutritional status in this study. No significant association was found between marital status and nutritional status, which was in consonance with results of Boulos et al. in Lebanon ${ }^{23}$ and Saikia and Mahanta in Guwahati. ${ }^{24}$ Smoking status and treatment funding had no association with nutritional status.

Loss of nutritional reserves due to alcohol ingestion is partly due to inadequate protein intake and is compounded by the effects that ethanol has on gastrointestinal function, which includes increased mucosal permeability which may lead to 'leakage' of nutrients from the blood to the gut lumen, increased gut motility with increased transit times, and impaired salt and water absorption. Alcohol also inhibits the absorption of vitamins and nutrients by active transport processes, an effect that may be crucial in precipitating specific nutrient deficiencies e.g. thiamine. The end result may be severe functional impairment and tissue damage in other organs, notably the liver and the brain, as a consequence of specific vitamin and nutrient deficiencies. ${ }^{25}$

In this study, the somatic risk factors like patients using walking aids, patients having urinary incontinence or urgency, patients using high fall risk drugs, patients experiencing a severe decline in food intake from last three months were significantly associated with nutritional status.

Malnutrition is a multifactorial condition. This study reported that the mobility functions, ${ }^{26-29}$ decline in food intake ${ }^{26,29}$ and weight loss ${ }^{26,27}$ are the major contributing risk factors of malnourishment. This study also reports that patients requiring walking aids contribute malnutrition mostly followed by patients receiving more than 6 medications, patients receiving high fall risk drugs (HFRDs), patients with urinary incontinence, patients having 3 or more comorbidities, and patients experienced fall within 6 months before admission, while evaluating the somatic risk factors of malnutrition.

This study has various strengths which include; it involves the most vulnerable age group "greater or equal to 60 years" which is very challenging to address and evaluate. It also provides a detailed insight regarding risk factors especially somatic risk factors of malnutrition and prevalence in general. There is a paucity of data available in this domain and these findings could run as a forerunner in the development of appropriate guidelines for nutritional screening and interventional programs among the geriatric population. The small sample size could be one of the major limitations of this study.

\section{Conclusion}

This study was aimed to evaluate the nutritional status, different risk factors associated with malnutrition and the influence of different determinants on the nutritional status among elderly patients at a tertiary care public teaching hospital by using MNA- SF tool. Analysis, using MNA-SF tool, showed that 118 patients were at "risk of malnutrition" and 109 patients were found to be "malnourished". The "at risk of malnutrition" group was the largest contributor (50.2\%). Mobility functions followed by a decline in food intake, weight loss, psychological stress/acute disease, neuropsychological problems and 
body mass index were identified risk factors in this study. Among somatic risk factors of malnutrition, patients required walking aids contribute malnutrition mostly followed by patients receiving more than 6 medications, patients receiving high fall risk drugs, patients with urinary incontinence, patients having 3 or more comorbidities, and patients experienced fall within 6 months before admission.

\section{Declaration of conflicting interests}

The authors declared no potential conflicts of interest with respect to the research, authorship, and/or publication of this article.

Funding

No external funding

\section{Authors contribution}

Conceived and design of the study: IR, PT, SSL.

Acquisition of data: IR.

Analysis and interpretation of data: IR, PT.

Drafting the manuscript: IR, PT.

Revising the manuscript critically for more intellectual content: IR, PT, SSL.

\section{Acknowledgment}

The authors thank the officers of "Government Medical College and Hospital, Chandigarh" and "National Institute of Pharmaceutical Education and Research, SAS Nagar India" for catalyzing the present study. The authors would extend the thanks to the developer of "Mini Nutritional Assessment-short form" Tool.

\section{Appendix A. Supplementary data}

Supplementary data to this article can be found online at https:// doi.org/10.1016/j.cegh.2019.05.001.

\section{References}

1. Villafane JH, Pirali C, Dughi S, et al. Association between malnutrition and Barthel Index in a cohort of hospitalized older adults article information. J Phys Ther Sci. 2016;28(2):607-612.

2. Schrader E, Baumgärtel C, Gueldenzoph H, et al. Nutritional status according to Mini Nutritional Assessment is related to functional status in geriatric patients-independent of health status. J Nutr Health Aging. 2014;18:257-263.

3. Mcwhirter JP, Pennington CR. Incidence and recognition of malnutrition in hospital. BMJ. 1994;308:945-948.

4. Hickson M. Malnutrition and ageing. Postgrad Med. 2006 Jan;82(963):2-8.

5. Gariballa SE, Parker SG, Taub N, et al. Nutritional status of hospitalized acute stroke patients. Br J Nutr. 1998;79:481-487.

6. Claggett MS. Nutritional factors relevant to Alzheimer's disease. J Am Diet Assoc. 1989;89:392-396.

7. Volicer L, Seltzer B, Rheaume Y, et al. Progression of Alzheimer-type dementia in institutionalised patients: a cross sectional study. J Appl Gerontol. 1987;6:83-94.

8. Blaum CS, Fries BE, Fiatarone MA. Factors associated with low body mass index and weight loss in nursing home residents. J Gerontol A Biol Sci Med Sci. 1995;50:M162-M168.

9. Thompson MP, Morris LK. Unexplained weight loss in the ambulatory elderly. J Am Geriatr Soc. 1991;39:497-500.

10. Westin T, Jansson A, Zenckert C, et al. Mental depression is associated with malnutrition in patients with head and neck cancer. Arch Otolaryngol Head Neck Surg. 1988;114:1449-1453.

11. Guigoz Y. The Mini Nutritional Assessment (MNA) review of the literature-what does it tell us? J Nutr Health Aging. 2006;10:466-485.

12. Kaiser MJ, Bauer JM, Uter W. Prospective validation of the modified mini nutritional assessment short-forms in the community, nursing home, and rehabilitation setting. $J$ Am Geriatr Soc. 2011;59:2124-2128.

13. Guigoz Y, Vellas B, Garry PJ. Mini nutritional assessment: a practical assessment tool for grading the nutritional state of elderly patients. Facts Res Gerontol. 1994(Suppl 2):15-59.

14. Forman DE, Berman AD, McCabe CH, Baim DS, Wei JY. PTCA in the elderly: the "young-old" versus the "old-old. J Am Geriatr Soc. 1992;40(1):19-22.

15. Vedantam A, Subramanian V, Rao NV, John KR. Malnutrition in free-living elderly in rural south India: prevalence and risk factors. Publ Health Nutr. 2010;13:1328-1332.

16. Agarwalla R, Saikia AM, Baruah R. Assessment of the nutritional status of the elderly and its correlates. J Fam Community Med. 2015;22:39-43.

17. Ferdous T, Kabir ZN, Wahlin A, Streatfield K, Cederholm T. The multidimensional background of malnutrition among rural older individuals in Bangladesh - a challenge for the Millennium Development Goal. Publ Health Nutr. 2009;12:2270-2278.

18. Saka B, Kaya O, Ozturk GB, Erten N, Karan MA. Malnutrition in the elderly and its relationship with other geriatric syndromes. Clin Nutr. 2010;29:745-748.

19. Anuradha S, Devina S, Neena M. A study on prevailing malnourishment among elderly population of Lucknow city. Int J Agric Food Sci Technol. 2014:5(2):35-40.

20. Saeidlou SN, Merdol TK, Mikaili P, Bektas Y. Assessment of the nutritional status and affecting factors of elderly people living at six nursing home in Urmia, Iran. Int J Acad Res. 2011;3:1.

21. Meena S, Singh VB, Meena BL, et al. Study of nutritional status in elderly in Indian population. Int J Current Res. 2014;6(11):10253-10257.

22. Ghani A, Hussain S, Zubair M. Assessment of nutritional status of geriatric population in Sargodha city. Int J med Appl health. 2013;1(1):22-29.

23. Boulos C, Salameh P, Barberger-Gateau P. The AMEL study, a cross sectional population-based survey on aging and malnutrition in 1200 elderly Lebanese living in rural settings: protocol and sample characteristics. BMC Public Health. 2013;13(1):573

24. Saikia A, Mahanta N. A study on nutritional status of elderly in terms of body mass index in Urban Slums of Guwahati City. J Ind Acad Geriatr. 2013;9:9-14.

25. World MJ, Ryle PR, Thomson AD. Alcoholic malnutrition and the small intestine. Alcohol Alcohol. 1985;20(2):89-124.

26. Shatenstein B, Kergoat MJ, Nadon S. Weight change, nutritional risk and its determinants among cognitively intact and demented elderly Canadians. Can J Public Health. 2001;92:143-149.

27. Mamhidir AG, Ljunggren G, Kihlgren M, Kihlgren A, Wimo A. Underweight, weight loss and related risk factors among older adults in sheltered housing-a Swedish follow-up study. J Nutr Health Aging. 2006;10:255-262.

28. Jyrkka J, Enlund H, Lavikainen P, Sulkava R, Hartikainen S. Association of polypharmacy with nutritional status, functional ability and cognitive capacity over a three-year period in an elderly population. Pharmacoepidemiol Drug Saf. 2011;20:514-522

29. Schilp J, Wijnhoven HA, Deeg DJ, Visser M. Early determinants for the development of undernutrition in an older general population: longitudinal Aging Study Amsterdam. Br J Nutr. 2011;106:708-717. 